\section{Real-time microscopic visualization of tissue response to laser thermal therapy}

\author{
Benjamin J. Vakoc, ${ }^{*}$ Guillermo J. Tearney, and \\ Brett E. Bouma \\ Harvard Medical School and Massachusetts General \\ Hospital, Wellman Center for Photomedicine, 55 Fruit Street, \\ BAR 703, Boston, Massachusetts 02114
}

\begin{abstract}
We present methods for visualizing the dynamic response of biological samples to laser-induced heating. Our approach utilizes optical frequency-domain imaging to detect, spatially localize, and monitor unique dynamic signatures that arise within zones of active tissue denaturation. Since this information is precisely registered with high-resolution $(\sim 10 \mu \mathrm{m})$ cross sectional images, regions of thermally destroyed tissue can be mapped in relation to pre-existing morphology. Using porcine esophageal specimens ex vivo, we demonstrate that the extent and evolution of laser thermal damage can be assessed in real time. () 2007 Society of Photo-Optical Instrumentation Engineers. [DOI: 10.1117/1.2714027]
\end{abstract}

Keywords: laser thermal therapy; tissue response; optical frequency domain; laser damage.

Paper 06295LR received Oct. 20, 2006; revised manuscript received Jan. 2, 2007; accepted for publication Jan. 2, 2007; published online Apr. 18, 2007.

The use of targeted laser energy to induce localized zones of thermal coagulation and necrosis has been investigated as an intervention for a broad spectrum of disease processes. Prior studies and current clinical practice, however, have applied laser therapy without guidance and with minimal, if any, feedback, resulting in highly variable outcomes that include both frequent recurrence due to insufficient treatment and complications associated with overly aggressive treatment. ${ }^{1}$ Improved efficacy and reduced complications might result from the integration of in situ monitoring techniques capable of indicating in real time the boundaries of the induced thermal coagulation zone relative to local anatomical and pathologic features.

Because optical coherence tomography ${ }^{2}$ (OCT) is capable of visualizing architectural features of epithelial tissues, it is well suited to define therapeutic target volumes in situ. However, complementary OCT-based therapy monitoring techniques of sufficient accuracy and sensitivity have not been demonstrated, limiting the utility of integrated OCT-guided therapeutic instruments. In this work, we present novel methods for performing OCT-based monitoring of thermal therapy. Unlike existing techniques that rely on images of blood flow, ${ }^{3}$ birefringence, ${ }^{4}$ or optical scattering ${ }^{5}$ acquired after treatment, the methods of this work utilize OCT measurements acquired during therapeutic laser exposure. From the dynamic properties of these signals, information about the physical and bio-

*Tel: 617-726-0695; E-mail: bvakoc@partners.org

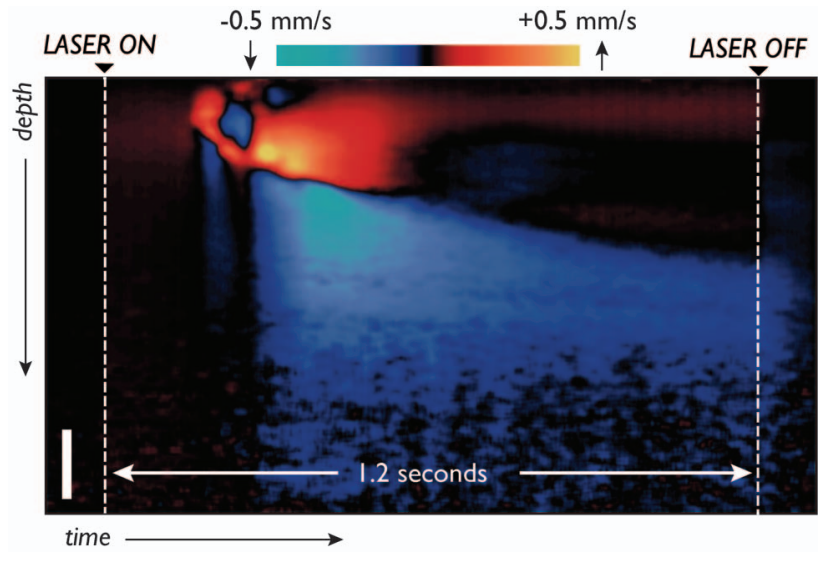

Fig. 1 An M-mode image generated using phase-sensitive processing during a 1.2-sec therapy beam exposure is shown.

mechanical changes that accompany the thermal coagulation process can be obtained. ${ }^{6}$ It is shown that, through this information, the boundary of an induced thermal injury can be measured in real time and with high resolution.

An optical frequency domain imaging (OFDI) system, ${ }^{7}$ a specific embodiment of recently developed frequency-domain OCT technology, was used to monitor the response of porcine esophageal tissue to laser thermal excitation ex vivo. In this study, an imaging A-line rate of $10 \mathrm{kHz}$ was used. The imaging beam was focused to a $23-\mu \mathrm{m}$ spot on the tissue surface. Freshly acquired porcine esophageal tissue samples were mounted between a fixed backing and a thin $(\sim 100 \mu \mathrm{m})$ glass coverslip. The use of esophageal tissue was motivated by the clinical application of laser therapy to the treatment of esophageal intramucosal cancer. Tissue samples were irradiated with $320 \mathrm{~mW}$ of $\mathrm{CW}$ power at $1450 \mathrm{~nm}$, a wavelength where absorption is dominated by water. The therapeutic beam was directed onto the tissue as a collimated beam with $1.1 \mathrm{~mm}$ diameter centered at the imaging beam focus and was gated by an optical switch that was synchronized to the OFDI system. This arrangement allowed the laser exposure to be registered in time and correlated with the measured OFDI data. OFDI data were continuously acquired in M-mode fashion without translating the sample, yielding depth-resolved measurements of the same axial line as a function of time during laser exposure.

Thermal injury evolution was monitored through analysis of the measured depth-resolved complex reflectivity $A(z, t)$. Two complementary methods of analysis were developed. Each method focused on separate dynamic properties of the measured complex reflectivity. In the first method, changes in the phase of the complex reflectivity were extracted through phase-resolved Doppler OFDI processing techniques. ${ }^{8}$ This phase-resolved approach sensitively measured the axial displacements that resulted from coagulation-induced tissue deformation processes. In the second method, variations in the magnitude of the complex reflectivity $\Delta|A|$ were extracted to detect relative changes in the magnitude and/or location of the optical scattering centers within the imaging resolution element. This magnitude variation approach contrasts with the

1083-3668/2007/12(2)/020501/3/\$25.00 @ 2007 SPIE 


\section{JBO LETTERS}

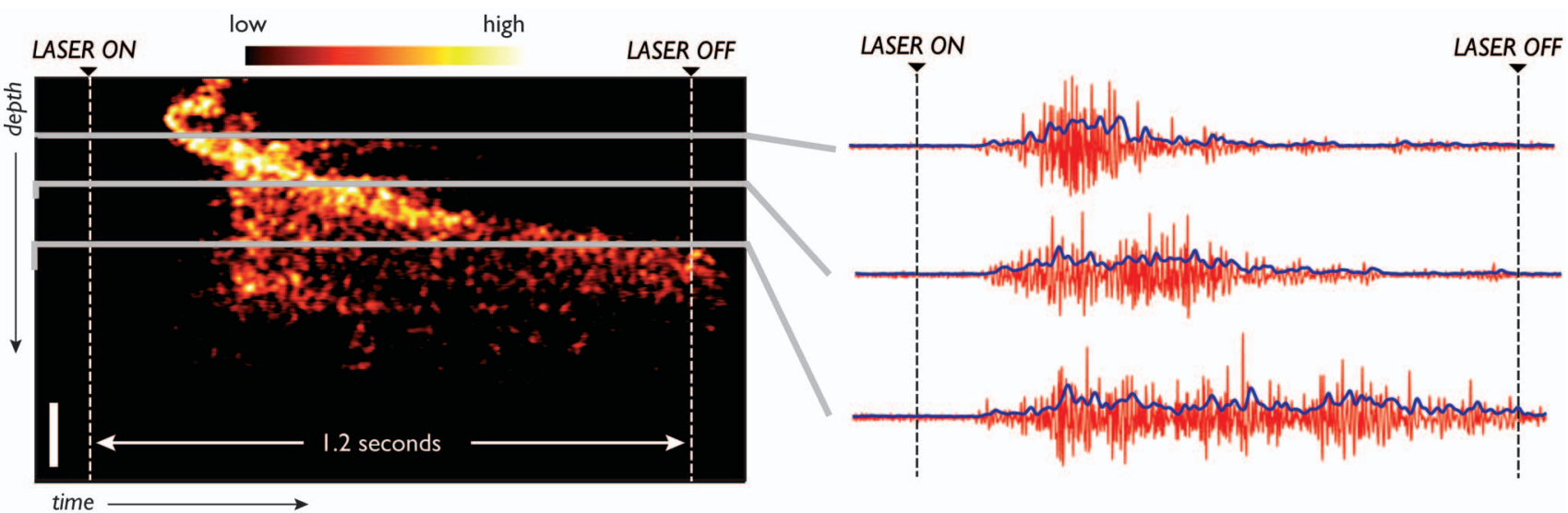

Fig. 2 An M-mode image generated using magnitude variation processing during a 1.2-sec therapy beam exposure is shown. The bandpass filtered amplitude signals (red traces) and their resulting demodulated power signals (blue traces) are presented for the three indicated depths to illustrate this approach.

phase measurements in which the overall aggregate changes of the scattering centers in the axial dimension comprise the basis of the signal. By performing these measurements as a function of time and depth, M-mode images that provide a visualization of the thermal therapy process can be generated.

Figures 1 and 2 present the results for both the phaseresolved method and magnitude variation method, respectively, for the case of a $1.2-\mathrm{sec} 320-\mathrm{mW}$ therapy beam exposure. In Fig. 1, the phase difference signal, measured as a function of depth and time, is presented as an M-mode image. The 1.2-sec exposure time of the therapy laser is indicated at the top of the image. OFDI Doppler processing was used to extract phase difference information with a depth resolution of $8 \mu \mathrm{m}$ (determined by the system axial resolution) and temporal resolution of $0.1 \mathrm{~ms}$ (determined by the system A-line rate). This information was averaged using a rectangular filter of size $35 \mu \mathrm{m}$ in depth by $20 \mathrm{~ms}$ in time to reduce noise, and the resulting phase differences were mapped to the indicated color space. Minimal phase response was observed during the first $0.4 \mathrm{sec}$ of therapeutic laser exposure. Subsequently, the phase measurement indicated a region of upward/stationary
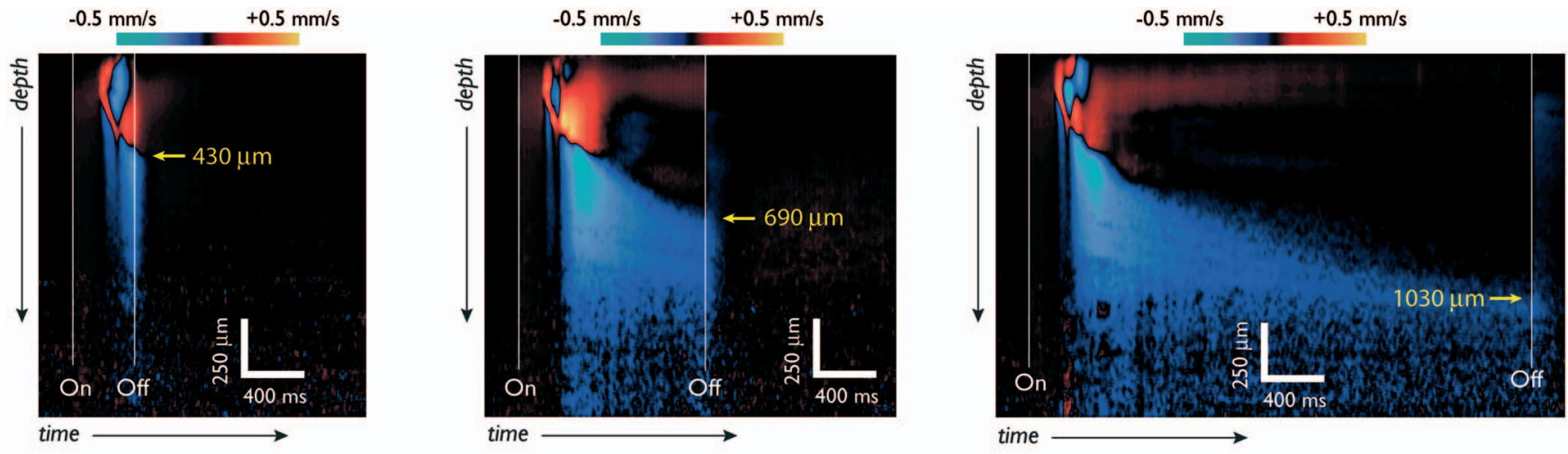

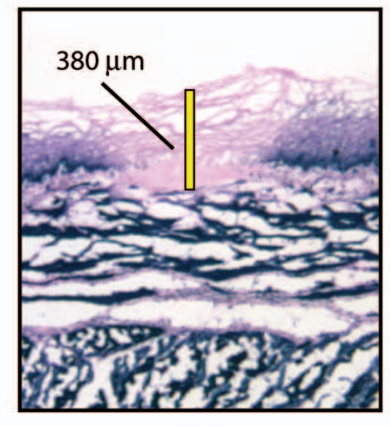

(a)

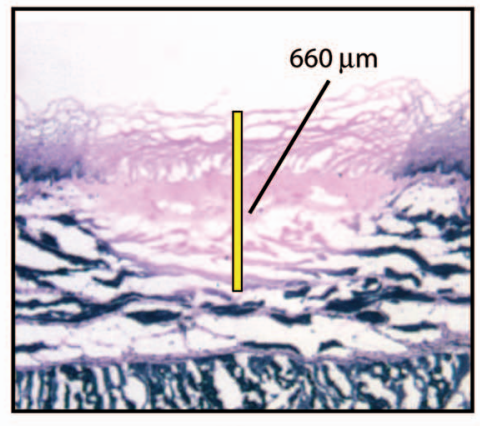

(b)

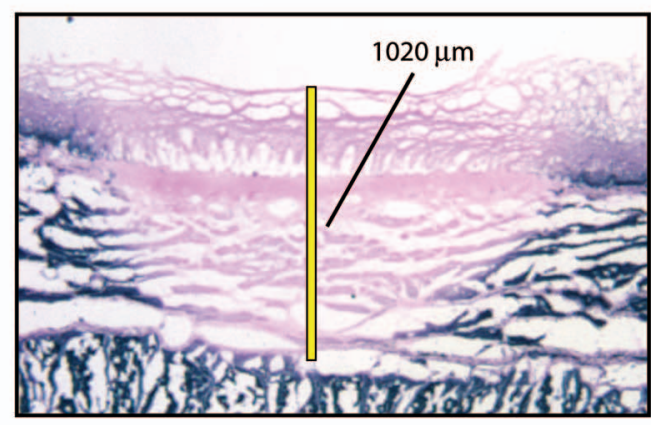

(c)

Fig. $3 \mathrm{M}$-mode images generated using the phase-sensitive processing method are shown for therapy beam exposure durations of (a) 0.4, (b) 1.2, and (c) $3.2 \mathrm{sec}$. Histology of the respective porcine esophageal tissue samples using NBTC staining is presented below each M-mode image. Note that the histologically determined injury depth matches the boundary of the downward deflected region (yellow arrow) in the M-mode images. 
deflection overlying a region of downward deflection. The boundary between these regions propagated downward monotonically during continued laser heating, and ceased promptly on termination of laser exposure. Our preliminary interpretation of this result is the following. On the initiation of laser exposure, a temperature distribution with a roughly exponential decay in depth begins to form. By $0.4 \mathrm{sec}$, the peak temperature exceeds the threshold of approximately $60{ }^{\circ} \mathrm{C}$ required to initiate protein denaturation, and the resulting local deformation gives rise to the observed phase difference signal. ${ }^{9}$ As laser delivery continues, the temperature distribution elevates and the isotherm corresponding to threshold propagates to deeper regions, resulting in a downward shift of active deformation and, hence, downward displacement of the zone of intense phase difference signal.

In Fig. 2, an M-mode image of the magnitude variation signal is presented, in which the depth-localized intensity of the signal magnitude fluctuations is mapped according to the indicated color bar. The following algorithm was developed to extract the magnitude variation signal. The log reflectivity magnitude, $\log [|A(z, t)|]$, was extracted at each depth as a function of time and digital bandpass [finite impulse response (FIR)] filtering was performed to extract the signal fluctuations within a passband from 100 to $500 \mathrm{~Hz}$. This frequency band was empirically determined to preferentially contain those fluctuations that are localized to the boundary between the coagulated and uncoagulated tissue regions, and thus they likely result from thermal coagulation processes. Figure 2 illustrates the bandpass filtered signals (red traces) and their corresponding demodulated power signal (blue traces) for the three indicated depths. The M-mode image was formed by mapping the demodulated power signals to the indicated color space. Unlike the phase-resolved approach illustrated in Fig. 1 , areas of high magnitude variation are localized within the boundary region that separates the thermally coagulated zone from the uncoagulated zone. This is consistent with the previously described intuition that the magnitude variation approach is sensitive to the reorganization of scattering centers during coagulation, a process that ceases after the tissue has thermally denatured. This approach appears to indicate regions undergoing coagulation, allowing delineation of uncoagulated (viable and untreated) regions from fully coagulated (treated) regions. Note that the boundary indicated by the magnitude variation method of Fig. 2 matches precisely the boundary between the upward and downward deflecting regions shown in the phase-resolved image of Fig. 1.

To assess the accuracy of these methods, measurements were acquired on separate esophageal tissue samples for a series of exposure durations. The resulting lesions were histologically processed using nitro-blue tetrazolium chloride (NBTC) staining, which labels the thermolabile enzyme lactate dehydrogenase (LDH). Prior studies have correlated loss of LDH activity with thermal injuries. ${ }^{10}$ Immediately after exposure, the tissue samples were frozen with the cover glass and backing in place. After freezing, the samples were re- moved from the mounting and submitted for histology. Figures 3(a)-3(c) illustrate the observed M-mode images of the phase-resolved approach for each of three exposure durations, along with the corresponding histology images. In each case, the laser power was set to $320 \mathrm{~mW}$. Loss of LDH activity in the histology is indicated by the absence of the blue precipitate. Measurements of injury depth were performed using the boundary of the LDH stain in the histology image (yellow bar) and the deepest location of the upward deflecting region in the M-mode images (yellow arrow). All measurements in OFDI were scaled assuming an index of refraction of 1.38. In all cases, the OFDI measurements of injury depth agreed with the histology.

In summary, we demonstrate a novel method for monitoring laser heating of biological tissue in real time. Although future studies will be required to further elucidate the physical mechanisms underlying the observed phase and intensity signals that arise during laser thermal treatment of tissue, the demonstrated correspondence with histologically determined injury depth suggests that these techniques may enable precise monitoring and mapping of laser thermal therapy.

\section{Acknowledgments}

This research was supported in part by the US National Institutes of Health (contract 1R21CA125560) and by the Center for Integration of Medicine and Innovative Technology (CIMIT).

\section{References}

1. M. H. Johnston, "Technology insight: ablative techniques for Barrett's esophagus-current and emerging trends," Nature Clinical Practice Gastroenterol. Hepatol. 2, 323-330 (2005).

2. D. Huang, E. A. Swanson, C. P. Lin, J. S. Schuman, W. G. Stinson, W. Chang, M. R. Hee, T. Flotte, K. Gregory, C. A. Puliafito, and J. G. Fujimoto, "Optical coherence tomography," Science 254, 1178 (1991).

3. J. K. Barton, A. J. Welch, and J. A. Izatt, "Investigating pulsed dye laser-blood vessel interaction with color Doppler optical coherence tomography," Opt. Express 3, 251-256 (1998).

4. J. F. De Boer, S. M. Srinivas, A. Malekafzali, Z. P. Chen, and J. S. Nelson, "Imaging thermally damaged tissue by polarization sensitive optical coherence tomography," Opt. Express 3, 212-218 (1998).

5. S. A. Boppart, J. Herrmann, C. Pitris, D. L. Stamper, M. E. Brezinski, and J. G. Fujimoto, "High-resolution optical coherence tomographyguided laser ablation of surgical tissue," J. Surg. Res. 82, 275-284 (1999).

6. J. F. Black and J. K. Barton, "Chemical and structural changes in blood undergoing laser photocoagulation," Photochem. Photobiol. 80, 89-97 (2004).

7. B. J. Vakoc, S. H. Yun, J. F. De Boer, G. J. Tearney, and B. E. Bouma, "Phase-resolved optical frequency domain imaging," Opt. Express 13, 5483-5493 (2005).

8. Y. Zhao, Z. Chen, C. Saxer, S. Xiang, J. F. De Boer, and J. S. Nelson, "Phase-resolved optical coherence tomography and optical Doppler tomography for imaging blood flow in human skin with fast scanning speed and high velocity sensitivity," Opt. Lett. 25, 114-116 (2000).

9. A. L. Mckenzie, "Physics of thermal processes in laser-tissue interations," Phys. Med. Biol. 35, 1175-1209 (1990).

10. R. A. Neumann, R. M. Knobler, F. Pieczkowski, and W. Gebhart, "Enzyme histochemical analysis of cell viability after argon laserinduced coagulation necrosis of the skin," J. Am. Acad. Dermatol. 25, 991-998 (1991). 